\title{
Thermopower of an SU(4) Kondo resonance under an SU(2) symmetry-breaking field
}

\author{
P. Roura-Bas ${ }^{1}$ L. Tosi ${ }^{2}$ A. A. Aligia,${ }^{2}$ and P. S. Cornaglia ${ }^{2}$ \\ ${ }^{1}$ Dpto de Física, Centro Atómico Constituyentes, \\ Comisión Nacional de Energía Atómica, Buenos Aires, Argentina \\ ${ }^{2}$ Centro Atómico Bariloche and Instituto Balseiro, \\ Comisión Nacional de Energía Atómica, 8400 Bariloche, Argentina
}

(Dated: July 28, 2018)

\begin{abstract}
We calculate the thermopower of a quantum dot described by two doublets hybridized with two degenerate bands of two conducting leads, conserving orbital (band) and spin quantum numbers, as a function of the temperature $T$ and a splitting $\delta$ of the quantum dot levels which breaks the SU(4) symmetry. The splitting can be regarded as a Zeeman (spin) or valley (orbital) splitting. We use the non-crossing approximation (NCA), the slave bosons in the mean-field approximation (SBMFA) and also the numerical renormalization group (NRG) for large $\delta$. The model describes transport through clean $\mathrm{C}$ nanotubes and in Si fin-type field effect transistors, under an applied magnetic field. The thermopower as a function of temperature $S(T)$ displays two dips that correspond to the energy scales given by the Kondo temperature $T_{K}$ and $\delta$ and one peak when $k_{B} T$ reaches the charge-transfer energy. These features are much more pronounced than the corresponding ones in the conductance, indicating that the thermopower is a more sensitive probe of the electronic structure at intermediate or high energies. At low temperatures $\left(T \ll T_{K}\right) T_{K} S(T) / T$ is a constant that increases strongly near the degeneracy point $\delta=0$. We find that the SBMFA fails to provide an accurate description of the thermopower for large $\delta$. Instead, a combination of Fermi liquid relations with the quantum-dot occupations calculated within the NCA gives reliable results for $T \ll T_{K}$.
\end{abstract}

PACS numbers: 72.20.Pa, 72.15.Qm,73.23.Hk, 73.63.Nm

\section{INTRODUCTION}

Materials with potentially useful thermoelectric properties and in particular large thermopower, are currently a subject of intense research due to their potential applications, for example in refrigeration or conversion of waste heat into electricity $\underline{\underline{1}} \underline{\underline{\underline{7}}}$ In addition, the thermopower is a useful tool to obtain additional insight on fundamental problems, like the Kondo effect $\underline{\underline{8}-11}$ The focus of research on thermal and thermoelectric properties has moved in the last years to nanostructures such as quantum dots, $\stackrel{11-16}{=}$ carbon nanotubes, $\stackrel{17,18}{=}$ molecules $\stackrel{19}{ }$ nanowires, and spin systems $\stackrel{20-23}{2}$ As we will show, the thermopower can be a more useful tool than the conductance, to study some features of the electronic structure of Kondo systems at finite energies.

Electronic and thermal transport through single level quantum dots is well described by the SU(2) Anderson model, and the Seebeck coefficient (thermopower) has been calculated using this model $11,14-16$ In particular, Costi and Zlatić have made a comprehensive study of the transport properties using the numerical renormalization group (NRG) $\stackrel{11}{=}$ A particularly interesting multilevel system is the SU(4) Anderson model, which describes quantum dots in carbon nanotubes, $\stackrel{24-29}{=}$ and silicon nanowires ${ }^{30}$ In contrast to the $\mathrm{SU}(2)$ case, the electron spectral density near the Fermi level of the SU(4) Anderson model in the strong coupling (Kondo) limit for nearly one electron at the dot is highly asymmetric, ${ }^{24,28}$ leading to a high derivative at the Fermi energy, which (neglecting phonon-drag effects) in turn is proportional to the Seebeck coefficient $S$ at low temperatures $\underline{11}$
The interest in Si nanowires increased due to the possibility of reducing the thermal conductance $\kappa$, leading to a large figure of merit $\sigma S^{2} T / \kappa$, where $\sigma$ is the conductivity $\underline{\underline{31}}$ Recently, the conductance of Si fin-type field effect transistors has been measured under an applied magnetic field $B$, which leads to a crossover from an $\mathrm{SU}(4)$ to an orbital SU(2) Kondo effect $\underline{30}$ The results were interpreted using the non-crossing approximation (NCA). In $\mathrm{C}$ nanotubes, even for $B=0$, there might be a symmetry reduction to $\mathrm{SU}(2)$ due to disorder-induced intervalley mixing, 29

In this work, we calculate the thermopower of the Anderson impurity model for two doublets, each one either spin or orbital degenerate, and infinite on-site Coulomb repulsion in the Kondo limit, as a function of the level splitting $\delta$, which corresponds to the Zeeman splitting for two orbitally degenerate levels in presence of an applied magnetic field, as in Si fin-type field effect transistors ${ }^{30}$ The symmetry of the model is $\mathrm{SU}(4)$ for $\delta=0$ and $\mathrm{SU}(2)$ for $\delta \neq 0$. We use complementary theoretical approaches: the NCA, $, 9,9,28$ slave bosons in the mean-field approximation (SBMFA) $\stackrel{32-35}{=}$ and Fermi liquid theory for low temperatures. We also use NRG to test the other approaches in the $\mathrm{SU}(2)$ limit.

The SBMFA satisfies Fermi liquid relationships at $T=0$ and is expected to capture the low-energy physics in the Kondo limit. In turn, while NCA has an error of about $15 \%$ in the value of the spectral density at very low temperature according to the Friedel sum rule $\stackrel{28}{2}$ it describes better the whole behavior, as shown for example in a previous comparison of NCA and NRG results in the $\mathrm{SU}(2)$ case ${ }^{36} \mathrm{In}$ addition, if the differential conductance $G$ is normalized at $T=0$, the leading behavior of $G$ for 
small voltage and temperature ${ }^{37}$ agrees with alternative Fermi liquid approaches,$\underline{38}-\underline{41}$ and the temperature dependence of the conductance practically coincides with the NRG result over several decades of temperature $\underline{37}$ Calculations of the thermopower for more than one spindegenerate level within the NCA, compared well with experiments in some Ce compounds $\stackrel{8.9}{*}$ NRG is a very accurate technique at low temperatures $\underline{42}$ However, for two bands, the Hilbert space is increased 16 times in each iteration, instead of 4 times for one band, making the technique much more demanding if the same degree of accuracy is wished. In addition, the SU(4) symmetry cannot be used to reduce the size of the matrices, since this symmetry is broken by $\delta$. Therefore we use here NRG only in the limit $\delta \rightarrow+\infty$, in which only one doublet and the band that mixes with it play a significant role.

The paper is organized as follows. In Section II we describe the model used. The approximations and the equation for the Seebeck coefficient are described in Section [III. The numerical results are presented in Section [V] Section $V]$ contains a summary and a short discussion.

\section{MODEL}

We start with a generalization of the Anderson model for infinite on-site Coulomb repulsion, which contains a singlet $|s\rangle$ with $\mathcal{N}$ (even) particles and two spin doublets $|i \sigma\rangle(i=1,2$ is the valley index; $\sigma=\uparrow$ or $\downarrow)$ with $\mathcal{N}+1$ (or $\mathcal{N}-1$ ) particles representing the four spin and valley degenerate states of a quantum dot created for example by depleting the density at two points of a $\mathrm{C}$ nanotube or in a Si nanowire. The dot is connected to two conducting leads which are also spin and valley degenerate. The $\mathrm{SU}(4)$ symmetry is then broken by applying a magnetic field or breaking the valley degeneracy in a simple way. In both cases, interchanging spin $(\sigma)$ and valley $(i)$ indices if necessary, the Hamiltonian can be written in the form

$$
\begin{aligned}
H= & E_{s}|s\rangle\left\langle s\left|+\sum_{i \sigma} E_{i}\right| i \sigma\right\rangle\langle i \sigma|+\sum_{\nu k \sigma} \epsilon_{\nu k} c_{\nu k i \sigma}^{\dagger} c_{\nu k i \sigma} \\
& +\sum_{i \nu k \sigma}\left(V_{\nu}|i \sigma\rangle\langle s| c_{\nu k i \sigma}+\text { H.c. }\right),
\end{aligned}
$$

where $c_{\nu k i \sigma}^{\dagger}$ create conduction states at the left $(\nu=L)$ or right $(\nu=R)$ lead, and $V_{\nu}$ is the hopping between the lead $\nu$ and both doublets, assumed independent of $k$ for simplicity. The doublets are split by an energy $\delta=E_{2}-E_{1}$. This corresponds to the Zeeman splitting when the $\mathrm{SU}(4)$ symmetry of the model for $\delta=0$ is broken by an applied magnetic field.

While there are four spin degenerate bands of mobile electrons, depending on valley index $i$ or position with respect to the quantum dot (left or right), for each energy $\epsilon_{L k}=\epsilon_{R k^{\prime}}$ for which there are states at the left and the right, only the linear combination $V_{L k} c_{L k i \sigma}^{\dagger}+V_{R k^{\prime}} c_{R k^{\prime} i \sigma}^{\dagger}$ hybridizes with the state $|i \sigma\rangle$. Thus, the model is effectively a two-band model.
We note that in the case of intervalley mixing induced by disorder in $\mathrm{C}$ nanotubes, the hopping elements for small magnetic field depend on the valley and lead indices $\frac{29}{2}$ In this case, the formalism used in this work is not applicable. It seems that a full non-equilibrium formalism is needed to treat the most general case, as that developed for the conductance in Ref. 43 and sketched in Ref. 44. The model is not applicable either for the case of magnetic impurities in $\mathrm{C}$ nanotubes, for which symmetry-breaking geometrical effects play an important role $\stackrel{45}{4}$

\section{THE FORMALISM}

\section{A. Equations for the thermopower}

In the limit of vanishing applied bias voltage and temperature difference between the leads, the electronic part of the transport coefficients can be evaluated in terms of the total spectral density at the $\operatorname{dot} \rho_{d}(\omega)=\sum_{i \sigma} \rho_{i \sigma}(\omega)$. This is possible due to the fact that the couplings between the quantum dot and the right or left leads are proportional. $\underline{46}$ If this were not the case (as in nanotubes affected by disorder $\underline{29}$ or for some molecules $\underline{43}$ ) a different formalism would be needed. $\stackrel{43,44}{4}$

The Seebeck coefficient is simply given by $\underline{\underline{11}}$

$$
S=\frac{-I_{1}(T)}{e T I_{0}(T)}
$$

where $e$ is the absolute value of the electronic charge and

$$
I_{n}=\int \omega^{n} \rho_{d}(\omega)\left(-\frac{\partial f}{\partial \omega}\right) d \omega
$$

where $f(\omega)$ is the Fermi function, and the zero of energy is taken at the Fermi energy $\epsilon_{F}=0$

\section{B. Approximations for the spectral density}

To calculate the total spectral density $\rho_{d}(\omega)$ that enters Eqs. (3) we have used mainly the NCA. At $T=0$ have used the SBMFA and also a combination of Fermi liquid relationships and quantum-dot occupations obtained using NCA. In the limit $\delta \rightarrow+\infty$, we have also used NRG to shed light on the virtues and shortcomings of the other approximations.

In the NCA and SBMFA, an auxiliary boson $b$, and four auxiliary fermions $f_{i \sigma}$ are introduced, so that the localized states are represented as

$$
|s\rangle=b^{\dagger}|0\rangle,|i \sigma\rangle=f_{i \sigma}^{\dagger}|0\rangle,
$$


where $|0\rangle$ is the vacuum. These pseudoparticles should satisfy the constraint

$$
b^{\dagger} b+\sum_{i \sigma} f_{i \sigma}^{\dagger} f_{i \sigma}=1 .
$$

The NCA solves a system of self-consistent equations to obtain the Green functions of the auxiliary particles, which is equivalent to sum an infinite series of diagrams (all those without crossings) in the corresponding perturbation series in the hopping, and afterwards a projection on the physical subspace of the constraint is made. The formalism of the NCA for this problem or similar ones is described in previous papers $\stackrel{8,9,24,43}{\text { In particu- }}$ lar, the more general case of complex hoppings is treated in Ref. 43. Therefore, we do not give more details here. The application of NRG in the case of only one doublet $(\delta \rightarrow+\infty)$ has also been explained before $\underline{\underline{11}}$

In the SBMFA, the boson operators are replaced by a number $b_{0}=\langle b\rangle$, and the energy is minimized with respect to $b_{0}$ and a Lagrange multiplier $\lambda$ that enforces Eq. (5). Assuming a constant density of unperturbed conduction electrons $\rho$ extending from $-D$ to $D$ and filled to the Fermi level $\epsilon_{F}=0$, a simple generalization of the case of one doublet,, 33.34 leads to the following change of energy after introduction of the impurity

$$
\begin{aligned}
E= & \frac{1}{\pi} \sum_{i}\left[\tilde{\Delta} \ln \left(\frac{\tilde{E}_{i}^{2}+\tilde{\Delta}^{2}}{D^{2}}\right)+2 \tilde{E}_{i} \arctan \left(\frac{\tilde{\Delta}}{\tilde{E}_{i}}\right)\right] \\
& -\frac{4 \tilde{\Delta}}{\pi}+\lambda\left(b_{0}^{2}-1\right),
\end{aligned}
$$

where $\tilde{E}_{i}=E_{i}+\lambda, \tilde{\Delta}=b_{0}^{2} \Delta$, and $\Delta=\pi \rho\left(V_{L}^{2}+V_{R}^{2}\right)$ is the total half resonant level width (adding the contributions from both leads). Above $D \gg \tilde{\Delta}$ was assumed. Minimizing $E$ with respect to $\lambda$ one obtains an equation that allows to relate $E_{1}$ (or $\lambda$ ) with half the quasiparticle level width $\tilde{\Delta}$ (which is of the order of the Kondo temperature). After some algebra we obtain

$$
\begin{aligned}
\tilde{E}_{1} & =\left[\frac{\delta^{2}}{4}+\left(1+\beta^{2}\right) \tilde{\Delta}^{2}\right]^{1 / 2}+\beta \tilde{\Delta}-\frac{\delta}{2}, \\
\beta^{-1} & =\tan \left[\frac{\pi}{2}\left(1-\frac{\tilde{\Delta}}{\Delta}\right)\right] .
\end{aligned}
$$

Minimizing $E$ with respect to $\tilde{\Delta}$ one obtains

$$
\frac{1}{\pi} \ln \frac{\left(\tilde{E}_{1}^{2}+\tilde{\Delta}^{2}\right)\left(\left(\tilde{E}_{1}+\delta\right)^{2}+\tilde{\Delta}^{2}\right)}{D^{4}}+\frac{\tilde{E}_{1}-E_{1}}{\Delta}=0,
$$

and replacing $\tilde{E}_{1}$ from Eq. (77) in Eq. (8) an equation for the single unknown $\tilde{\Delta}$ is obtained, which we solve numerically.

The occupation and the spectral density near the Fermi energy for each doublet are

$$
\begin{aligned}
n_{i \sigma} & =\langle\mid i \sigma\rangle\langle i \sigma \mid\rangle=\frac{1}{\pi} \arctan \left(\frac{\tilde{\Delta}}{\tilde{E}_{i}}\right), \\
\rho_{i \sigma}(\omega) & =\frac{b_{0}^{2} \tilde{\Delta} / \pi}{\left(\omega-\tilde{E}_{i}\right)^{2}+\tilde{\Delta}^{2}} .
\end{aligned}
$$

\section{Fermi liquid theory}

For an $\mathrm{SU}(\mathrm{N})$ model which channel index $j=1$ to $N$, and a simple symmetry breaking perturbation (like a generalized magnetic field) that does not mix channels, so that spin and channel are conserved, the Friedel sum rule relates the spectral density at the Fermi level $\epsilon_{F}$ with the number of displaced electrons for each channel $\stackrel{47}{*}$ The latter coincides with the occupation for a constant unperturbed density of conduction electrons with a wide band $D \gg \tilde{\Delta}$ as we assume, where $\tilde{\Delta}$ (of the order of the Kondo temperature $T_{K}$ ) is the resonant level width of the quasiparticles [as in the SBMFA, Eq. (9)]

Then one has 28,47

$$
\rho_{j}\left(\epsilon_{F}\right)=\frac{1}{\pi \Delta} \sin ^{2}\left(\pi n_{j}\right) .
$$

In addition, the derivatives at $\epsilon_{F}$ are also known (using for example renormalized perturbation theory) $)^{41,48,49}$,

$$
\left.\frac{\partial \rho_{j}(\omega)}{\partial \omega}\right|_{\epsilon_{F}}=\sin \left(2 \pi n_{j}\right) \frac{\rho_{j}\left(\epsilon_{F}\right)}{\tilde{\Delta}} .
$$

From Eqs. (9), it is apparent that the SBMFA (in which we have chosen $\epsilon_{F}=0$ ) satisfies these relationships.

Using Eqs. (10) and (11) and a Sommerfeld expansion in Eqs (3), the Seebeck coefficient Eq. (2) for $T \rightarrow 0$ becomes

$$
S=-\frac{2 \pi^{2} k_{B} T}{3 \tilde{\Delta}} \frac{\sum_{i \sigma} \sin ^{3}\left(\pi n_{i \sigma}\right) \cos \left(\pi n_{i \sigma}\right)}{\sum_{i \sigma} \sin ^{2}\left(\pi n_{i \sigma}\right)} .
$$

\section{NUMERICAL RESULTS}

Without loss of generality, we take $\epsilon_{F}=E_{s}=0$, where $\epsilon_{F}$ is the Fermi level of the leads. For the numerical calculations, we assume a constant density of states per spin of the leads $\rho=1 /(2 D)$ between $-D$ and $D$. We take the unit of energy as the total level width of both doublets $\Gamma=2 \Delta=2 \pi \rho\left(V_{L}^{2}+V_{R}^{2}\right)$. The energy of both doublets is denoted as $E_{1}=E_{d}, E_{2}=E_{d}+\delta$. 


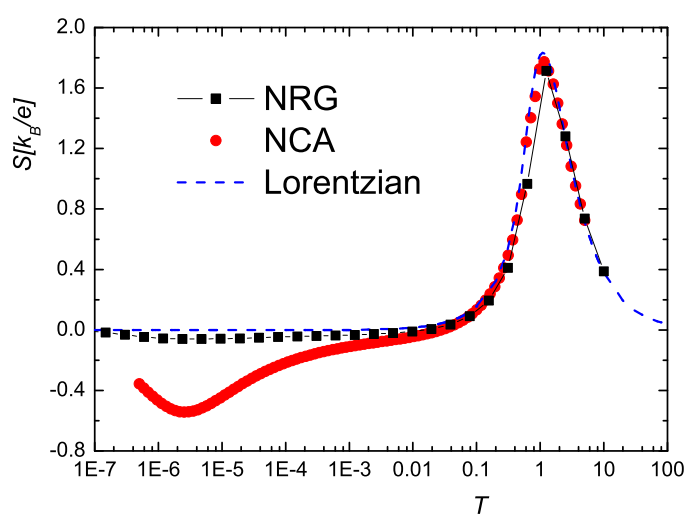

FIG. 1. (Color online) Thermopower as a function of temperature for $E_{d}=-4 \Gamma$ and $\delta \rightarrow+\infty$. Squares: NRG, dots: NCA, dashed line: Lorentzian spectral density (see text).

\section{A. The limit of one doublet}

For $\delta \rightarrow+\infty$, the model is equivalent to the limit of infinite on-site repulsion $U$ of the simplest Anderson model, studied in detail before using the NRG ${ }^{11}$ Here we compare results obtained with NRG with those of NCA and SBMFA to see the limitations of the different methods, which will be useful for the analysis of the general case.

In Fig. 11 we display the thermopower as a function of temperature in the Kondo regime. There is a dip near the Kondo temperature $T_{K}$ due to the fact that the Kondo peak in the spectral density is slightly above the Fermi level $\epsilon_{F}$, and a peak near the charge-transfer energy $\epsilon_{F}-$ $E_{d}$ due to the corresponding peak below $\epsilon_{F}$. We mean "near" as an estimation of the order of magnitude. For example, the maximum of the curve within NCA is at $T=1.10 \Gamma$, while $\epsilon_{F}-E_{d}=4 \Gamma$ in the figure.

The spectral density $\rho_{d}(\omega)$ of the Anderson model for only one doublet $(\delta \rightarrow+\infty)$ is well known. In particular, using a local-moment approach, Logan and coworkers have shown that in the Kondo regime, the charge-transfer peaks (which carry most of the spectral weight in this regime) have a Lorentzian shape of a width two times larger than that of the non-interacting case. 50 We have included in Fig. 1 the thermopower that results replacing $\rho_{d}(\omega)$ in Eqs. (2) and (3), by a simple Lorentzian of total width $2 \Gamma$ centered at $E_{d}$. The agreement with the NCA result at high temperatures is remarkable. Instead, the charge-transfer peak in the thermopower within NRG has a small shift to higher temperatures and an intensity about $10 \%$ lower. We have verified that this also happens for other values of $E_{d}$. This is probably related with resolution problems of the NRG at large energies, as discussed below.

At low temperatures, the NRG results are more reliable than those of NCA and SBMFA. We have extracted
TABLE I. Kondo temperature from $G(T)\left(T_{K}^{G}\right)$, total occupation of the lowest doublet $(n)$, linear coefficient of $S(T)$ from NRG $\left(S T_{K}^{G} / T\right)$ and from a Fermi liquid theory $(S \tilde{\Delta} / T)$ for different values of the charge-transfer energy $\epsilon_{F}-E_{d}$.

\begin{tabular}{||l||l|l|l|l||}
\hline \hline$E_{d} / \Gamma$ & $T_{K}^{G} / \Gamma$ & $n$ & $S T_{K}^{G} / T$ & $S \tilde{\Delta} / T$ \\
\hline \hline-1 & $2.22 \times 10^{-2}$ & 0.732 & -3.48 & -2.450 \\
\hline-2 & $6.15 \times 10^{-4}$ & 0.897 & -0.93 & -1.047 \\
\hline-3 & $2.22 \times 10^{-5}$ & 0.941 & -0.51 & -0.611 \\
\hline-4 & $8.38 \times 10^{-7}$ & 0.958 & -0.38 & -0.437 \\
\hline-5 & $3.16 \times 10^{-8}$ & 0.967 & -0.27 & -0.339 \\
\hline-6 & $1.18 \times 10^{-9}$ & 0.973 & -0.22 & -0.317 \\
\hline \hline
\end{tabular}

the linear term in $T$ in the Seebeck coefficient, plotting $S / T$ vs $T$ for small $T$ and looking at the extrapolation to $T=0$. The results are shown in Table I. They have an uncertainty of the order of $10 \%$. We have also calculated the conductance $G(T)$ (not shown) and the total occupancy $n$ adding both spins. Using the results for $G(T)$, we estimated the Kondo temperature from the requirement that $G\left(T_{K}^{G}\right)=G(0) / 2$. Using $n_{1 \uparrow}=n_{1 \downarrow}=n / 2$, $n_{2 \uparrow}=n_{2 \downarrow}=0$, and the Fermi liquid expression Eq. (12), $S / T$ is calculated in an independent way. Taking into account the exponential variation of $T_{K}$ with $E_{d}$ one can see a semiquantitative agreement between both results in Table 1. The remaining quantitative discrepancy can be ascribed to the difference between $T_{K}^{G}$ and $\tilde{\Delta}$ as a measure of the Kondo temperature. In fact from renormalized perturbation theory one obtains $T_{K}^{G} / \tilde{\Delta}=0.746$ in the extreme Kondo limit while this ratio increases beyond 1 when valence fluctuations are allowed,, 39 and the Wilson ratio decreases $\underline{38-41}$

The NCA has the drawback that it does not fulfill Fermi liquid relationships. For example, the spectral density at the Fermi energy $\rho_{d}(0)$ at temperatures well below $T_{K}$ differs by about 10 or $20 \%$ in the Kondo regime from the value predicted by the Friedel sum rule. ${ }^{28} \mathrm{~A}$ detailed comparison of $\rho_{d}(\omega)$ between NRG and NCA in the Kondo regime is given by Fig. 10 of Ref. 36. One can see that in addition to the larger value of $\rho_{d}(0)$, the spectral density is more asymmetric for the NCA. This is probably the main reason of the factor near five between the magnitude of the dip in $S(T)$ near $T_{K}$ calculated with NCA with respect to the NRG result. Part of the discrepancy is probably also due to lack of resolution at finite energies within the NRG. For example in models of two quantum dots, the split Kondo peaks in the spectral density are considerably broadened, losing intensity (Fig. 11 of Ref. 51). Another example is the plateau in the conductance $G(T)$ observed at intermediate temperatures $T$ in transport through $\mathrm{C}_{60}$ molecules for gate voltages for which triplet states are important, $, 52,53$ which was missed in early NRG studies, but captured by the NCA 54,55 More recent NRG calculations using tricks to improve the resolution,, 56 have confirmed this plateau ${ }^{53}$

In any case, the one-level $\mathrm{SU}(2)$ limit is the worst case 


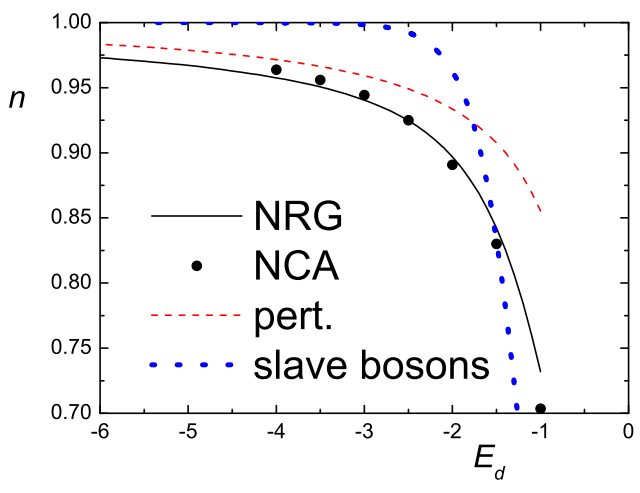

FIG. 2. (Color online) Total occupancy as a function of $E_{d}$ for $\delta \rightarrow+\infty$ and different approximations.

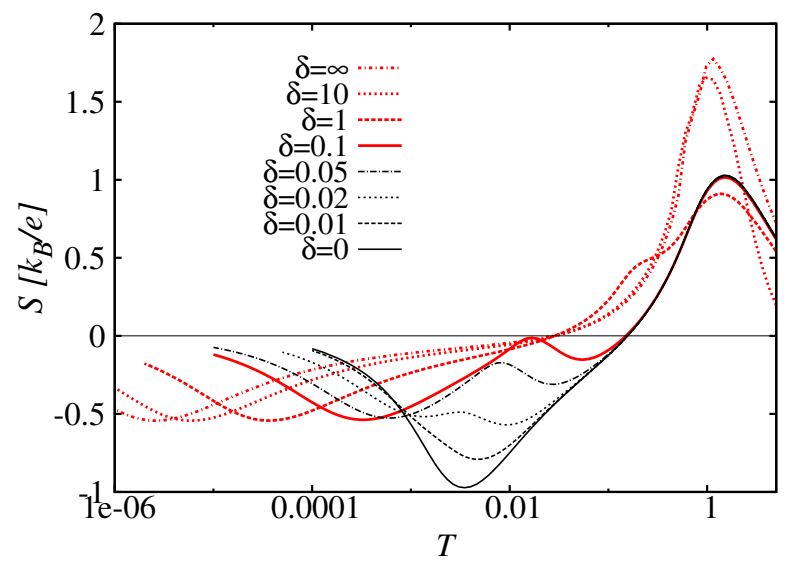

FIG. 3. (Color online) Thermopower as a function of temperature for $E_{d}=-4 \Gamma$ and different values of the level splitting.

for the NCA, because the real spectral density tends to be symmetric, while the NCA improves with increasing $\mathrm{N}$ for $\mathrm{SU}(\mathrm{N})$ models.$\stackrel{8}{ }$

The absolute value of $S$ is exaggerated within NCA for $T \rightarrow 0$. However, as shown in Fig. 2, there is a good agreement between the occupancies calculated with NRG and NCA. This suggests that using the occupancies calculated with NCA and Eq. (12) a semiquantitatively correct result for the linear part of $S(T)$ as $T \rightarrow 0$ can be obtained. Instead, while the SBMFA satisfies Fermi liquid relationships, the occupancies are not accurate for $\delta \rightarrow+\infty$. Even perturbation theory up to second order in $V_{\nu}$ neglecting spin flip leads to a better result for $n$, although (in contrast to SBMFA) this approach is unable to predict the right magnitude of $T_{K}$.

\section{B. Temperature dependence in the general case}

In Fig. 3 we represent the NCA results for the Seebeck coefficient $S(T)$ as a function of temperature for differ- ent values of $\delta$, which represents the splitting due to a Zeeman term for example. The spectral density $\rho_{d}(\omega)$ for finite $\delta$ has been studied before $\stackrel{24.28}{ }$ At $\delta=0, \rho_{d}(\omega)$ has a peak just above the Fermi energy $\epsilon_{F}$ with width of the order of $2 T_{K}^{4}$, where $T_{K}^{4}$ is the Kondo temperature of the $\mathrm{SU}(4)$ limit $\delta=0$. For the parameters of Fig. 3, $T_{K}^{4}$ is of the order of $0.01 \Gamma \stackrel{28}{2}$ As $\delta$ increases above $T_{K}^{4}$, the Kondo peak splits and another peak at an energy of the order of $\delta$ above $\epsilon_{F}$ appears. This peak above $\epsilon_{F}$ originates a dip in $S(T)$ for $T \sim \delta$. Note that peaks in $\rho_{d}(\omega)$ above $\epsilon_{F}$ lead to a positive contribution to $I_{1}$ [see Eq. (3)], which in turn lead to a negative contribution to $S(T)$ at the corresponding temperature [see Eq. (2)] Most of the spectral weight of the spectral density lies in the charge-transfer peak at energy $E_{d}$, which lies below the Fermi energy. Thus, when the temperature reaches values of the order of the charge-transfer energy $\epsilon_{F}-E_{d}$, the thermopower becomes positive $\left[I_{0}>0, I_{1}<0\right.$ in Eq. (2)].

As discussed above, for $\delta \rightarrow+\infty$ (the case of one $\mathrm{SU}(2)$ doublet), $S(T)$ shows one dip at $T_{K} \sim 10^{-6} \Gamma$ and a peak near the charge-transfer energy. For $\delta=0$, these qualitative features remain, but the Kondo temperature is four orders of magnitude larger, and the dip near $T_{K}^{4}$ is more pronounced, due to the larger asymmetry of the peak

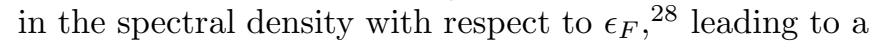
larger integral $I_{1}$ [see Eqs. (2) and (3)]. Since $T_{K}$ changes with $\delta$, as discussed below, the dip at $T_{K}$ displaces towards larger temperatures as $\delta$ decreases. Instead, the charge-transfer peak remains approximately at the same temperature and decreases in magnitude due mainly to additional broadening of the corresponding peak in the spectral density $\rho(\omega)$ as the $\mathrm{SU}(4)$ limit is approached, and also due to some transfer of the spectral weight to the Kondo peak. While this transfer is not large, the Kondo peak has a larger weight in the integrals $I_{n}$ due to the factor of the derivative of the Fermi function [see Eqs. (2) and (3)].

For $\delta>T_{K}^{4}$, for example $\delta=0.02 \Gamma$ in Fig. 3, an additional dip develops due to the peak near $\delta$ in $\rho_{d}(\omega)$. As $\delta$ increases further, the dip moves to higher temperatures, as it is apparent for $\delta=0.05$ and 0.1 in the figure. The relative minimum of $S(T)$ near the dip lies at temperatures of the order of $\delta$ but smaller, probably because of the large intensity of the peak near the charge-transfer energy, which pushes this minimum to lower temperatures. For $\delta=1$ this dip turns to a shoulder at the left of the charge-transfer peak and for larger $\delta$, the dip and the peak cross, interchanging the order of temperatures for which they appear.

In Fig. 4 we show the thermopower as a function of $T / T_{K}(\delta)$, where $T_{K}(\delta)$ is the Kondo temperature for each value of $\delta$. Here we determined $T_{K}(\delta)$ from the requirement that the conductance $G\left(T_{K}(\delta)\right)=e^{2} / h$ for symmetric leads $\left(V_{L}=V_{R}\right)$. To a good degree of accuracy, it 


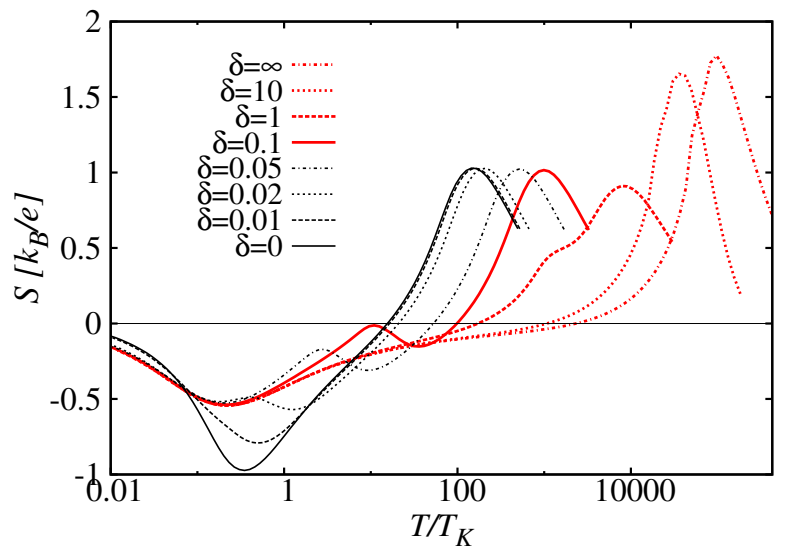

FIG. 4. (Color online) Thermopower as a function of $T / T_{K}$ for $E_{d}=-4 \Gamma$ and different values of the level splitting.

is given by the expression

$$
T_{K}(\delta)=\left\{(D+\delta) D \exp \left[\frac{\pi E_{1}}{2 \Delta}\right]+\frac{\delta^{2}}{4}\right\}^{1 / 2}-\frac{\delta}{2},
$$

obtained from a simple variational wave function. 28,44 With increasing $\delta, T_{K}$ stays roughly constant until $\delta>$ $T_{K}^{4}=T_{K}(0)$ and then it decreases strongly. From Fig. 4 it is apparent that the dip at smaller temperatures remains at $T \sim T_{K}(\delta)$ for all values of $\delta$. One can also see that the magnitude of the dip and (the absolute value of the thermopower for $T \sim T_{K}$ ) increases as $\delta$ decreases, being maximum at the $\mathrm{SU}(4)$ point $\delta=0$. Note that while the magnitude of this dip is exaggerated by the NCA for $\delta \rightarrow+\infty$, as explained in the previous section, we believe that this is not the case for $\delta=0$, because the spectral density is naturally asymmetric in this case, and since the NCA is a $1 / \mathrm{N}$ expansion, its accuracy improves with $\mathrm{N}$ in $\mathrm{SU}(\mathrm{N})$ models. $\stackrel{8}{-}$ Furthermore NCA calculations of the thermopower of Ce compounds, in which orbital degeneracy is important, compared well with experiment $\underline{8.9}$

In Fig. 5 we show the conductance $G(T)$ calculated with the NCA for different values of $\delta . G(0)$ is slightly larger for $\delta \rightarrow+\infty$ and lower for $\delta=0$ with respect to the correct values due to the failure of the NCA spectral density to satisfy Friedel sum rule ${ }^{28}$ In spite of this shortcoming, the overall shape and temperature dependence of $G$ in these limits agree with those obtained using NRG $: 11,25$

The point that we want to stress here is that although the finite energy features for $T \sim \delta$ and $\epsilon_{F}-E_{d}$ are present not only in $S(T)$ but also in $G(T)$, they are much weaker in $G(T)$. Thus, the Seebeck coefficient might be the appropriate tool to study the electronic structure of the system at intermediate energies.

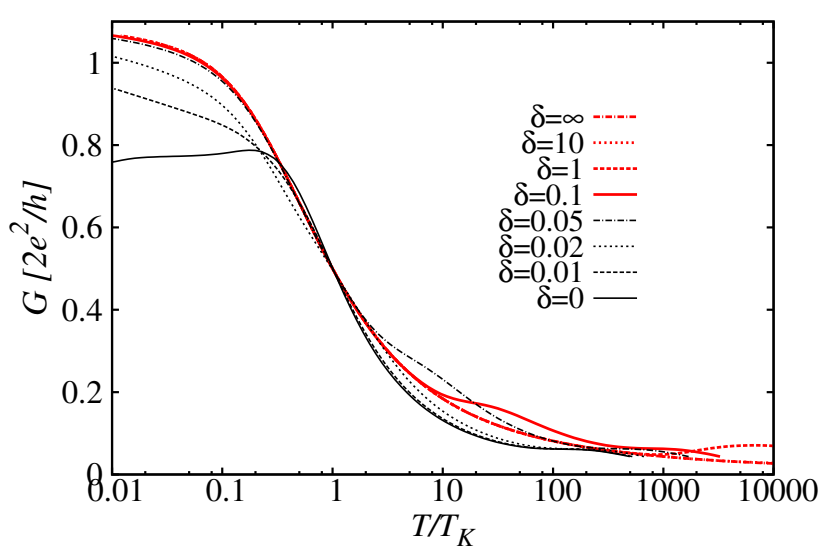

FIG. 5. (Color online) Conductance as a function of $T / T_{K}$ for $E_{d}=-4 \Gamma$ and different values of the level splitting.

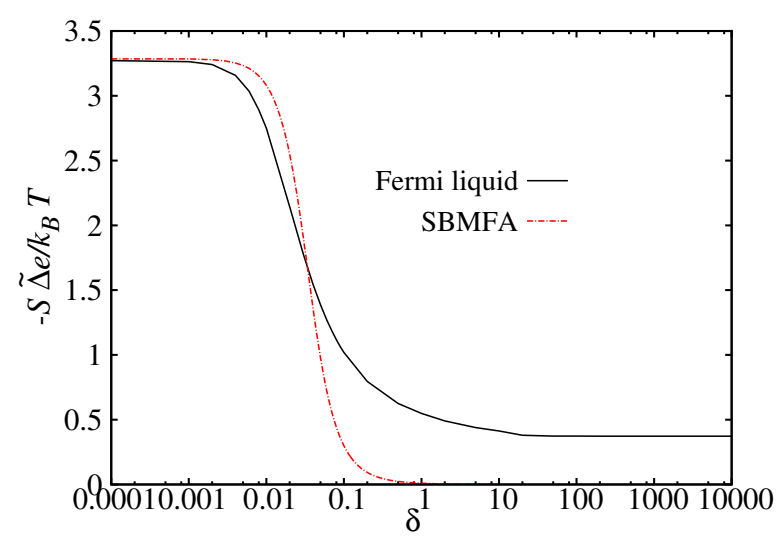

FIG. 6. (Color online) Coefficient of the linear dependence of the thermopower as a function of the level splitting, for $E_{d}=-4 \Gamma$.

\section{Dependence on splitting for $T \rightarrow 0$}

In this subsection, we present results for the term linear in temperature $T$ of the thermopower, as $T \rightarrow 0$, using two techniques: SBMFA and the Fermi liquid expression Eq. (12) with occupations calculated with the NCA. In Fig. [6 we represent $-S / T$ as a function of $\delta$. In the $\mathrm{SU}(4)$ limit $\delta=0$, both techniques agree and indicate a very large absolute value of $S \tilde{\Delta} / T$. In fact, in the extreme Kondo limit of an $\mathrm{SU}(\mathrm{N})$ model, one has $n_{j}=$ $1 / \mathrm{N}$ and from Eqs. (2), (3), (10), (11) and a Sommerfeld expansion one obtains

$$
S=-\frac{\pi^{2} T}{3 \tilde{\Delta}} \sin (2 \pi / N)
$$

and $|S \tilde{\Delta} / T|$ reaches its maximum value $\pi^{2} / 3=3.29$ for $\mathrm{N}=4$. The value in Fig. 6 is slightly smaller due to some degree of intermediate valence.

As $\delta$ increases, there is little variation until the Kondo temperature of the $\mathrm{SU}(4)$ limit $T_{K}^{4}(\sim 0.01 \Gamma$ in the figure) 


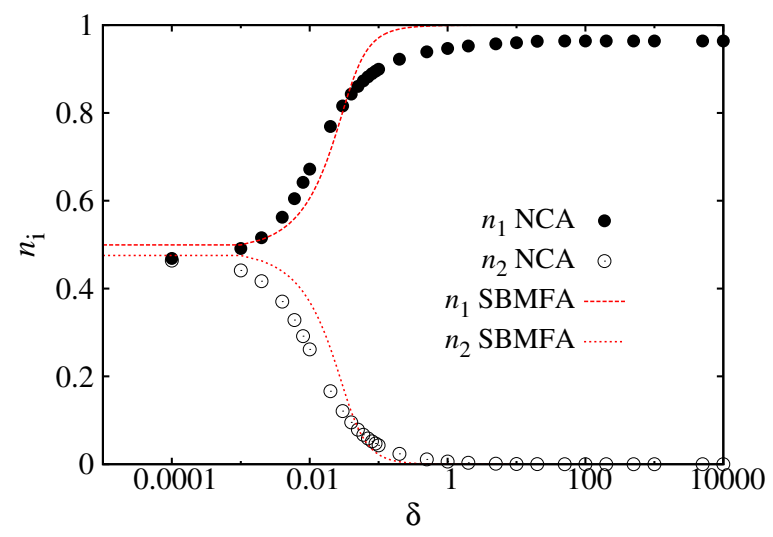

FIG. 7. (Color online) Occupation of the two doublets $n_{i}=$ $\sum_{\sigma} n_{i \sigma}$ as a function of the level splitting, for $E_{d}=-4 \Gamma$.

is reached. For larger $\delta, S \tilde{\Delta} / T$ falls due to the change of occupations (see Fig. 7): the lower doublet becomes more populated, while the occupation of the higher one decreases, keeping a total occupation slightly below 1 . While the trend of the curve is the same for both approaches, NCA and SBMFA, the absolute value of the thermopower decreases too much within the SBMFA for $\delta>10 T_{K}^{4}$. This is due to the fact discussed in Section IVA that the occupation predicted by the SBMFA of the lower lying doublet is too large for $\delta \rightarrow+\infty$. While this shortcoming affects the conductance or thermodynamic properties in a few \%, the effect of this increase is more dramatic in the thermopower.

Instead, since the NCA occupations agree with NRG in the limit $\delta \rightarrow+\infty$, the approach that combines NCA occupations and Fermi liquid relationships is reliable in this limit. The agreement with SBMFA and general expectations for the SU(4) model in the Kondo limit, indicates that this approach is also reliable for $\delta=0$.

\section{SUMMARY AND DISCUSSION}

We have calculated the thermopower of a model that describes electronic transport through quantum dots in $\mathrm{C}$ nanotubes, in which the disorder does not play an essential role, and Si fin-type field effect transistors in the Kondo regime. This regime can be easily controlled by applying a gate voltage that modifies the energy of the localized levels $E_{d}$. Without disorder and applied magnetic field, the model has SU(4) symmetry, as explained in Section I. In this case, the thermopower as a function of temperature $S(T)$ has a dip (negative $S$ ) at temperatures near the Kondo temperature $T_{K}$, and a peak (positive $S$ ) at temperatures near the charge-transfer energy.
For $\mathrm{SU}(\mathrm{N})$ symmetry, varying $\mathrm{N}$ with width of the Kondo resonance $\sim 2 T_{K}$ fixed, $\mathrm{N}=4$ is the most favorable case to have a large thermopower at temperatures lower that the Kondo temperature.

When the SU(4) symmetry is broken, leading to an energy splitting $\delta$ between two $\mathrm{SU}(2)$ doublets, by a simple symmetry breaking field (like a magnetic field), a new dip appears in $S(T)$ at temperatures of the order of $\delta$. While this dip the peak for positive $S$ at the charge-transfer energy are clearly displayed in $S(T)$, the corresponding features in the conductance $G(T)$ at equilibrium are very weak. This suggest that the study of the thermopower might be a more useful tool to study the electronic structure at finite energies. Another alternative is to study the conductance out of equilibrium from which peaks at a bias voltage of the order of $\pm \delta / e$ are expected 30,44

As $\delta$ increases, the characteristic energy scale $T_{K}(\delta)$, which determines among several physical scales, the width of the Kondo resonance and the temperature at which $G(T)$ falls to half its value at $T=0$, decreases following a simple expression Eq. (13). For $T \ll T_{K}(\delta)$, $S$ is linear in $T$ with a coefficient that stays approximately constant for $\delta<T_{K}(0)$. As $\delta$ increases further, $S T_{K}(\delta) / T$ for $T \rightarrow 0$ decreases by an order of magnitude but $T_{K}(\delta)$ decreases by nearly three orders of magnitude, so that the linear coefficient increases. For this calculation, an approach that combines Fermi liquid relationships with occupation numbers calculated with $\mathrm{NCA}$ gives more reliable results that the SBMFA.

Because of the nature of the underlying SU(4) symmetry, the couplings of the four states involved to the conducting leads are proportional, and independent of the state ( $V_{L}$ to the left and $V_{R}$ to the right), This fact which simplifies the calculations is no more true for weak mag-

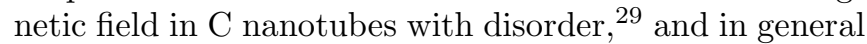
in multilevel quantum dots $\frac{57}{.}$ In these cases, as well as in situations with total or partial destructive interference, such as transport through molecules, $\stackrel{43,44,58}{\underline{4}}$ the present formalism does not apply and a full non-equilibrium formalism seems necessary $\underline{\underline{43}}$ In addition here, the total occupation of the dot is below 1 , in contrast to models with multilevel systems in which even occupation is allowed ${ }^{53}$

\section{ACKNOWLEDGMENTS}

We thank CONICET from Argentina for financial support. This work was partially supported by PIP 11220080101821 of CONICET and PICT R1776 of the ANPCyT, Argentina. P. R. is sponsored by Escuela de Ciencia y Tecnología, Universidad Nacional de San Martín.
1 T. M. Tritt, Annu. Rev. Mater. Res. 41, 433 (2011).
2 B. C. Sales, D. Mandrus, and R. K. Williams, Science 272, 1325 (1996). 
3 I. Terasaki, Y. Sasago, and K. Uchinokura, Phys. Rev. B 56, R12685 (1997).

4 A. Bentien, S. Johnsen, G. K. H. Madsen, B. B. Iversen, and F. Steglich, EPL 80, 17008 (2007).

5 M. G. Kanatzidis, Chem. Mater. 22, 648 (2010).

${ }^{6}$ R. F. Klie, Q. Qiao, T. Paulauskas, A. Gulec, A. Rebola, S. Ögüt, M. P. Prange, J. C. Idrobo, S. T. Pantelides, S. Kolesnik, B. Dabrowski, M. Ozdemir, C. Boyraz, D. Mazumdar, and A. Gupta, Phys. Rev. Lett. 108, 196601 (2012).

7 G. D. Mahan, and J. O. Sofo, Proc. Natl. Acad. Sci. USA, 93, 7436 (1996).

8 N. E. Bickers, D. L. Cox, and J. W. Wilkins, Phys. Rev. B 36, 2036 (1987).

${ }^{9}$ V. Zlatić and R. Monnier, Phys. Rev. B 71, 165109 (2005).

10 T. A. Costi, A. C. Hewson, and V. Zlatić, J. Phys.: Condens. Matter 6, 2519 (1994); T. A. Costi and A. C. Hewson, ibid. 5, L361 (1993).

11 T. A. Costi and V. Zlatić, Phys. Rev. B 81, 235127 (2010).

12 T. C. Harman, P. J. Taylor, M. P. Walsh, and B. E. Laforge, Science 297, 2229 (2002).

13 R. Scheibner, H. Buhmann, D. Reuter, M. N. Kiselev, and L. W. Molenkamp, Phys. Rev. Lett. 95, 176602 (2005).

14 D. Boese and R. Fazio, Europhys. Lett. 56, 576 (2001).

15 B. Dong and X. L. Lei, J. Phys.: Condens. Matter 14, 11747 (2002).

16 T.-S. Kim and S. Hershfield, Phys. Rev. Lett. 88, 136601 (2002).

17 J. Hone, I. Ellwood, M. Muno, A. Mizel, M. L. Cohen, A. Zettl, A. G. Rinzler, and R. E. Smalley, Phys. Rev. Lett. 80, 1042 (1998).

18 C. H.Yu; L. Shi, Z. Yao, D. Y. Li and A. Majumdar, A. Nano Lett. 5, 1842 (2005).

19 P. Reddy, S-Y. Jang, R. A. Segalman, and A. Majumdar, Science 315, 1568 (2007).

20 A. V. Sologubenko, E. Felder, K. Giannò, H. R. Ott, A. Vietkine, and A. Revcolevschi, Phys. Rev. B 62, R6108 (2000).

21 A. V. Sologubenko, H. R. Ott, G. Dhalenne, and A. Revcolevschi, Europhys. Lett. 62, 540 (2003).

22 A. V. Rozhkov and A. L. Chernyshev, Phys. Rev. Lett. 94, 087201 (2005).

${ }^{23}$ L. Arrachea, G. S. Lozano, and A. A. Aligia, Phys. Rev. B 80, 014425 (2009).

24 J. S. Lim, M.-S. Choi, M. Y. Choi, R. López, and R. Aguado, Phys. Rev. B 74, 205119 (2006).

${ }^{25}$ F. B. Anders, D. E. Logan, M. R. Galpin, and G. Finkelstein, Phys. Rev. Lett. 100, 086809 (2008).

26 S. Lipinski and D. Krychowski, Phys. Rev. B 81, 115327 (2010).

27 C. A. Büsser, E. Vernek, P. Orellana, G. A. Lara, E. H. Kim, A. E. Feiguin, E. V. Anda, and G. B. Martins, Phys. Rev. B 83, 125404 (2011).

28 L. Tosi, P. Roura-Bas, and A. A. Aligia, Physica B 407, 3259 (2012).

29 K. Grove-Rasmussen, S. Grap, J. Paaske, K. Flensberg, S. Andergassen, V. Meden, H. I. Jorgensen, K. Muraki, and
T. Fujisawa, Phys. Rev. Lett. 108, 176802 (2012).

30 G. C. Tettamanzi, J. Verduijn, G. P. Lansbergen, M. Blaauboer, M. J. Calderón, R. Aguado, and S. Rogge, Phys. Rev. Lett. 108, 046803 (2012).

31 Y. He and G. Galli, Phys. Rev. Lett. 108, 215901 (2012).

32 P. Coleman, Phys. Rev. B 29, 3035 (1984).

33 A. C. Hewson, The Kondo Problem to Heavy Fermions, Cambridge University Press, Cambridge, England, 1993.

34 A. A. Aligia and L. A. Salguero, Phys. Rev. B 70, 075307 (2004); Phys. Rev. B 71, 169903(E).

35 A. M. Lobos and A. A. Aligia, Phys. Rev. Lett. 100, 016803 (2008); Physica B 404, 3306 (2009).

36 T. A. Costi, J. Kroha and P. Wölfle, Phys. Rev. B 53, 1850 (1996).

37 P. Roura-Bas, Phys. Rev. B 81, 155327 (2010).

38 A. Oguri, J. Phys. Soc. Jpn. 74, 110 (2005).

39 J. Rincón, A. A. Aligia, and K. Hallberg, Phys. Rev. B 79, 121301(R) (2009); Phys. Rev. B 80, 079902(E) (2009); Phys. Rev. B 81, 039901(E) (2010).

40 E. Sela and J. Malecki, Phys. Rev. B 80, 233103 (2009).

41 A. A. Aligia, J. Phys. Condens. Matter 24, 015306 (2012); references therein.

42 R. Bulla, A.C. Hewson, and Th. Pruschke, J. Phys. Cond. Matt. 10, 8365 (1998).

43 L. Tosi, P. Roura-Bas, and A. A. Aligia, J. Phys. Condens. Matter 24, 365301 (2012).

44 P. Roura-Bas, L. Tosi, A. A. Aligia, and K. Hallberg, Phys. Rev. B 84, 073406 (2011).

45 P. P. Baruselli, A. Smogunov, M. Fabrizio, and E. Tosatti, Phys. Rev. Lett. 108, 206807 (2012).

46 Y. Meir and N. S. Wingreen, Phys. Rev. Lett. 68, 2512 (1992).

47 A Yoshimori and A Zawadowski, J. Phys. C 15, 5241 (1982).

48 A. Yoshimori, Prog. Theor. Phys. 55, 67 (1976).

49 S. Kirchner, J. Kroha, and P. Wölfle, Phys. Rev. B 70, 165102 (2004).

50 D. E. Logan, M. P. Eastwood and M. A. Tusch, J. Phys.: Condens. Matter 10, 2673 (1998).

51 L. Vaugier, A.A. Aligia and A.M. Lobos, Phys. Rev. B 76, 165112 (2007).

52 N. Roch, S. Florens, V. Bouchiat, W. Wernsdorfer, and F. Balestro, Nature 453, 633 (2008).

53 S. Florens, A, Freyn, N. Roch, W. Wernsdorfer, F. Balestro, P. Roura-Bas and A. A. Aligia, J. Phys. Condens. Matter 23, 243202 (2011); references therein.

54 P. Roura-Bas and A. A. Aligia, Phys. Rev. B 80, 035308 (2009).

55 P. Roura-Bas and A. A. Aligia, J. Phys. Cond. Matt. 22, 025602 (2010).

56 A. Freyn and S. Florens, Phys. Rev. Lett. 107, 017201 (2011).

57 W. Izumida, O. Sakai, and Y. Shimizu, J. Phys. Soc. Jpn. 67, 2444 (1998).

58 J. Rincón, K. Hallberg, A. A. Aligia, and S. Ramasesha, Phys. Rev. Lett. 103, 266807 (2009); references therin. 